\title{
INFLUENCE OF MANUFACTURING QUALITY OF LAMINATED CORE ON A TURBOGENERATOR EXPLOITATION TERM
}

\begin{abstract}
The problem of calculating the flexural deformation of the laminated core of the stator turbogenerator is examined. The evaluation of the effect of gluing the core sheets on its bending stiffness based on the compliance of the insulating cover sheet of active steel. An impact of pressing effort on the relative movement of the package of sheets is determined. An effect of wear of the insulating layer between sheets of laminated core on its reliability is determined. Conclusions are drawn on how to determine the possibility of extending the life of turbogenerators on the basis of the data obtained. References 8, table 1, figures 5.

Key words: laminated core, flexural deformation, stator of turbogenerator, flexural stiffness, insulation coating, pressing, time of exploitation.
\end{abstract}

Рассмотрен расчет изгибной деформации иихтованного сердечника статора турбогенератора. Выполнена оценка влияния склейки листов сердечника на его изгибную жесткость с учетом податливости изоляционного покрытия листов активной стали, значения усилия прессовки пакета на относительное перемещение листов. Определено влияние износа изоляционного слоя между листами шихтованного сердечника на его надежность. Сделаны выводы, как на основании полученных данных установить возможсность продления срока эксплуатации турбогенераторов. Библ. 8, табл. 1, рис. 5 .

Ключевые слова: шихтованный сердечник, изгибная деформация, статор турбогенератора, изгибная жесткость, изоляционное покрытие, прессовка, время эксплуатации

Introduction. The economic condition of the country makes it impossible to perform the timely replacement of electrical equipment, which has worked on the technical specification operational time of the plant unit. Therefore, the period of its work can be extended only by means of modernization and/or partial replacement of major components. Modernization of turbogenerators (TG) is provided with the increase power in the installed overall dimensions, sometimes performed with replacement of refrigerant. Therefore, in the construction to be upgraded it is necessary to consider the accumulated experience of manufacturing and operation, to exclude all of the factors that lead to the destruction of the assembly machines and units, especially, a stator core, for reliability. Its destructions are the most frequent cause of crashes.

TG reliability and durability are reduced due to a complex laminated core structure, vibration, electromagnetic continuous variables, thermal and mechanical loads. Due to the frequent destruction of the cores the researchers are looking for new design solutions for increasing operational reliability TG. The new design is based on the use of new materials and new techniques in the production of technology, on the knowledge of data exploitation and modern trends of development of the theory of the creation of turbogenerators [1-3].

The aim of the article is to determine the effect of TG stator core assembly technology to its reliability and the definition of the possibility extending the service life (impact gluing the core sheets on its bending stiffness, impact of pressing forces on the relative movement of the package sheet and wear of the insulating layer between sheets of the laminated core).

The main text of the article. The design quality of the laminated stator core determines its bending stiffness and consequently affects the value of the radial vibration and reliability. The works of many authors are devoted to these issues, [4-8], which also show the results of the research physical and mechanical properties of the insulating cover sheet steel. Also, the temperature coefficients of the materials that are used, static and dynamic loads; the presence, size, shape of ventilation ducts between packets impact on it.

Consider the impact on the operational status of the core circuit stacking the stacked sheets and the quality of insulation coatings under the action of forces that cause the flexural deformation and the relative movement of the stacked sheets. Let us allocate part of the laminated core package, where several floors (joints) fall. We represent this part as a straight girder, which is made of rectangular thick electrical steel sheets with a thickness $h$ and isolated coatings $\delta$, Fig. 1 .

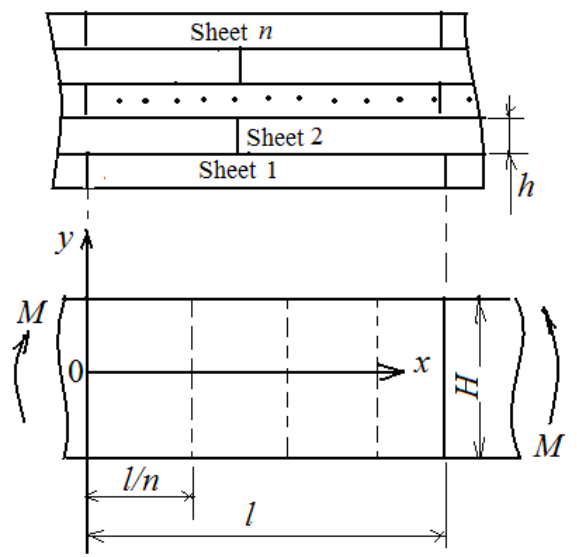

Fig. 1. Laminated veneer lumber: $h$ - thickness of the sheets constituting the laminated board (package of the stacked plates); $M$ - moment of bending forces; $H$-width of the beam; $l$ - length of the considered area (wave height); $n$ - number the joints, got in area under consideration

We believe in the calculations that the tension in all the sheets is identical. We obtain under the law of pairing shear stresses, that tangential stresses in the cross section are shear stresses, which arise in the longitudinal sections. It is suffice to consider the deformation of the strip in $n$ layers, when the sheets are stacked to overlap by $1 / n$ part of their length, because in the other layers of pattern the same will happen. 
Use Zhuravsky formula for further calculations, which allows you to determine the tangential stresses in bending. These stresses arise from the external force $P$ in the points of girder cross section, which are at a distance $l$ from the neutral axis $x$, Fig. 2. In the beam appear normal and tangent stresses, which are directed from the edge to the edge of the law paired relationship.

We calculate the shear stresses that occur. Spend two verticals through the beam cross section at a distance $d x$ from one another. Let us note a horizontal section at a distance $(h / 2-y)$ of the neutral layer. That is, we select the element with the size: $d x,(h / 2-y), b$.

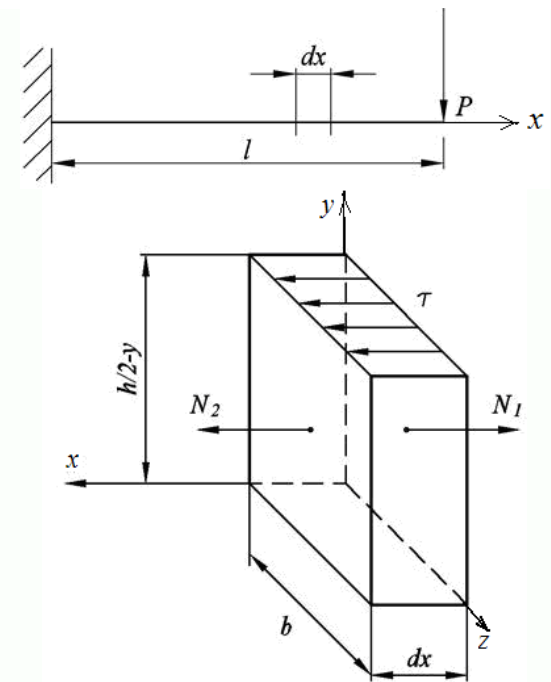

Fig. 2. Model actions of on a girder of the external force $F$, which is located at a distance $l$ from the neutral axis $x$

We lay off on the axis $x$ all the forces that act on the system for drawing up the balance equations. The action of elementary tangential force $d T=N_{2}-N_{1}$, which is equal to the element $(b \cdot d x)$, is equal to $d T=\tau \cdot b \cdot d x$, is balanced by the difference of normal forces $\left(N_{2}-N_{1}\right)$. Normal stresses that act on the infinitely small side area $d F$, disposed at the neutral axis $y$ can be calculated:

$$
\sigma=\frac{M \cdot y}{J_{x}} .
$$

The force $d N_{1}$ that acts on the area element is equal to:

$$
d N_{1}=\frac{M \cdot y}{J_{z}} \cdot d F .
$$

On the whole side face of area $F$ the aggregate force of $N_{1}$ will operate:

$$
N_{1}=\int_{F} d N_{1}=\int_{F} \frac{M \cdot y}{J_{z}} \cdot d F=\frac{M}{J_{z}} \cdot \int_{F} y \cdot d F=\frac{M \cdot S_{z}}{J_{z}},
$$

where $S_{z}$ - distribution of the tangential stresses a relatively the neutral axis $z ; J_{z}$ - axial moment inertia of the regarded section.

Similarly, the force that acts on the next side face of the selected element $N_{2}$ :

$$
N_{2}=\frac{(M+d M) \cdot S_{z}}{J_{z}},
$$

where $N_{2}-N_{1}=d T=\frac{d M \cdot S_{z}}{J_{z}}=\tau \cdot b \cdot d x$.

We write down the value of tangential stress $\tau$ :

$$
\tau=\frac{d M \cdot S_{z}}{d x \cdot J_{z} \cdot b}=\frac{Q \cdot S_{z}}{J_{z} \cdot b},
$$

where $Q$ - transverse elemental force of the bending moment that acts on the length of the fixed element $(Q=d M / d x)$.

The tangential stresses that arise in the material above the neutral layer is directly proportional to the transverse force, to the static moments square section under consideration, and is inversely proportional to the axial moment of inertia and its width. Then the distribution of tangential stresses over the cross section will be:

$$
\begin{aligned}
& S_{z}=F \cdot y_{0}=b \cdot\left(\frac{h}{2}-y\right) \cdot\left(y+\frac{\frac{h}{2}-y}{2}\right)= \\
& =\frac{b}{2} \cdot\left(\frac{h}{2}-y\right) \cdot\left(\frac{h}{2}+y\right)=\frac{b}{2} \cdot\left[\left(\frac{h}{2}\right)^{2}-y^{2}\right] .
\end{aligned}
$$

Substitute the $S_{z}$ in the formula (1). Define a tangential stress in a selected element based on the fact that the axial moment of inertia for the rectangular cross section is

$$
\begin{aligned}
& J_{z}=\frac{b \cdot h^{3}}{12}: \\
& \tau=\frac{\frac{b}{2} \cdot\left[\left(\frac{h}{2}\right)^{2}-y^{2}\right] \cdot Q}{\frac{b \cdot h^{3}}{12} \cdot b}=\frac{6 \cdot Q}{b \cdot h^{3}} \cdot\left(\frac{h^{2}}{4}-y^{2}\right) .
\end{aligned}
$$

The tangential stresses on the central axis with $y=0$ will be a half time more than average values in the sections, Fig. 3.

$$
\tau_{\max }=\frac{6 \cdot Q}{b \cdot h^{3}} \cdot \frac{h^{2}}{4}=\frac{3}{2} \cdot \frac{Q}{b \cdot h}=\frac{3}{2} \cdot \frac{Q}{F}=\frac{3}{2} \cdot \tau_{\text {mid }} .
$$

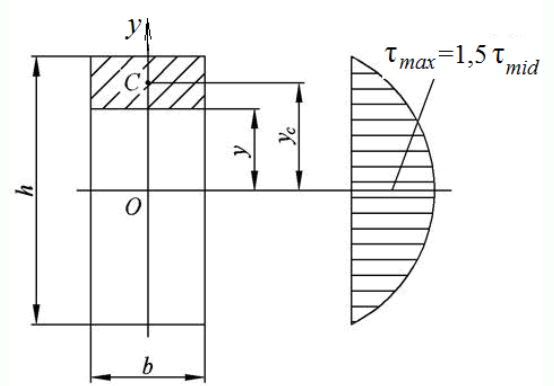

Fig. 3. The distribution of tangential stresses

The tangential stresses are the maximum value in the neutral layer; the normal stresses are equal to zero. So the tangential stresses are on the surface of the beam, only normal. It is believed that the transverse carved by area are level and are rotated by the action of the bending moments. But the tangential stresses distort the form of the deformation. Elements of the material between the two cross sections warp the proportion to the magnitude of these stresses. These distortions effect a little on the value of longitudinal deformation of the elements that make up the beam. This allows the use of a formula for the normal stresses:

$$
\sigma_{\max }=\frac{M_{u s e}^{\max }}{W_{z}} \leq[\sigma] \text {. }
$$


From beams of rectangular cross-section (Fig. 4,a) an element of length $d z$ is cut out (Fig. $4, b$ ).
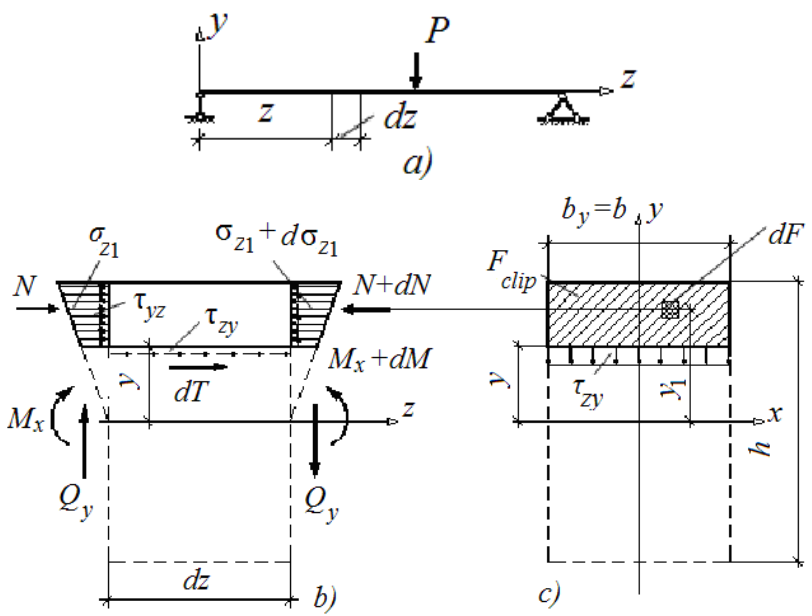

Fig. 4. The scheme of calculating tangential stresses in bending beam

Consider the balance of the top of the beams section, where stresses arise due to the difference of bending moments. In order this portion of the beam is in equilibrium $(\Sigma Z=0)$, in a longitudinal section of the beam there must be the tangential force $d T$.

The equilibrium equation of the girder:

$$
\begin{aligned}
& \Sigma Z=\int_{F_{\text {clip }}} \sigma_{\mathrm{z} 1} \cdot d F-\int_{F_{\text {clip }}}\left(\sigma_{\mathrm{z} 1}+d \sigma_{z 1}\right) \cdot d F+d T=0 . \\
& \text { Here } \\
& d T=\int_{F_{\text {clip }}} d \sigma_{z 1} \cdot d F=\int_{F_{\text {clip }}} \frac{d M_{x}}{I_{x}} \cdot y_{1} \cdot d F=\frac{d M_{x}}{I_{x}} \cdot S_{x}^{\text {clip }},
\end{aligned}
$$

where the integration is performed only over the severed part of the cross sectional area of $F_{\text {clip }}$ beams (the shaded area in Fig. 4,c); $S_{x}^{\text {clip }}$ - static moment of inertia of cross sectional area $d F$ of about the neutral axis $x$ :

$$
S_{x}^{\text {clip }}=\int_{F_{\text {clip }}} y_{1} \cdot d F
$$

Assume that the tangential stress $\left(\tau_{y z}\right)$, which arises in longitudinal section beams, distributed uniformly over its width $\left(b_{y}\right)$. Then:

$$
d T=\tau_{y z} \cdot b_{y} \cdot d z .
$$

Considering that $\tau_{y z}=\tau_{z y}$, the tangential stress $\left(\tau_{z y}\right)$ in the points in the cross section beams at a distance $y$ from the neutral axis $x$, can be calculated:

$$
\tau_{y z}=\frac{d M_{x}}{d z} \cdot \frac{S_{x}^{c l i p}}{I_{x} \cdot b_{y}}, \text { where } \frac{d M_{x}}{d z}=Q_{y}, \tau_{z y}=\frac{Q_{y} \cdot S_{x}^{c l i p}}{I_{x} \cdot b_{y}} .
$$

Longitudinal strain and stress have a major importance in the flexural deformation in a solid and glued laminated tool bar, as shown in [8]. We can assume that the tangential stress and normal stresses from deformation along the other axes are missing: $\sigma_{y}=\sigma_{z}=\tau_{x y}=\tau_{x z}=\tau_{y z}=0$.

We believe that the core layers operate only shear and that the longitudinal displacement of points $r_{i}(i=1,2, \ldots$ where $n$-number of the layer) on each sheet of thickness are the same. We obtain from the equilibrium conditions for the laminated section [7]:

$$
\left.\begin{array}{l}
E \cdot h \cdot \frac{\partial^{2} R_{1}}{\partial x^{2}}=\frac{G}{\delta} \cdot\left(2 R_{1}-R_{2}-R_{n}\right) ; \\
E \cdot h \cdot \frac{\partial^{2} R_{2}}{\partial x^{2}}=\frac{G}{\delta} \cdot\left(2 R_{2}-R_{1}-R_{3}\right) ; \\
E \cdot h \cdot \frac{\partial^{2} R_{i}}{\partial x^{2}}=\frac{G}{\delta} \cdot\left(2 R_{i}-R_{i-1}-R_{i+1}\right) ; \\
E \cdot h \cdot \frac{\partial^{2} R_{n}}{\partial x^{2}}=\frac{G}{\delta} \cdot\left(2 R_{n}-R_{n-1}-R_{1}\right),
\end{array}\right\}
$$

where $E$ - effective elastic modulus for the electrical steel sheet in the longitudinal direction (Unge modulus), $E=2 \cdot 10^{5} \mathrm{MPa} ; G$ - the shear modulus in the stacked structure (in the «glued layer»), $G=79,3 \cdot 10^{3} \mathrm{MPa}$.

We shall seek solution of the system of equations (2) as the dependence:

$$
R_{i}=A_{i}(y) \cdot e^{\lambda x}
$$

where $A_{i}(y)$ - the amplitude of the movement of the stacked structure along the axis $y ; \lambda-$ a constant which depends on the material (first Lame parameter).

For a particular material $\lambda$ expressed by Unge modulus $E$ and Poisson's ratio $v$ :

$$
\lambda=\frac{v \cdot E}{(1+v) \cdot(1-2 v)} .
$$

After a few solution transformations the following can be represented:

$$
R_{i}=A_{i 0}+B_{i 0} \cdot x+\sum_{k=1}^{n-1}\left(A_{i k} \cdot e^{\lambda_{k} \cdot x}+B_{i k} \cdot e^{-\lambda_{k} \cdot x}\right),
$$

where $A_{i k}(y)$ and $B_{i k}(y)$ - the function-coordinates of points along the y axis $y$ in a quantity $2 n^{2}$.

The expressions for $A_{i \kappa}$ and $B_{i \kappa}$ present with the use of the coefficient $B_{0}$ :

$$
A_{i \kappa}=B_{0} \cdot a_{k} ; \quad B_{i \kappa}=B_{0} \cdot b_{k},
$$

where $a_{k}$ and $b_{k}$ - the numerical coefficients.

The solution of system (4) can finally be written:

$$
\begin{aligned}
& R_{i}=A_{0}(y)+B_{0}(y) \cdot x+ \\
& +B_{0}(y) \cdot \sum_{k=1}^{n-1} a_{i k} \cdot\left(a_{k} \cdot e^{\lambda_{k} \cdot x}+b_{k} \cdot e^{-\lambda_{k} \cdot x}\right) .
\end{aligned}
$$

The average cross-section of each sheet is not corrected due to the symmetry of the tensions distribution. Therefore, we can write for the case of even $n$ :

$$
R_{n / 2}(0, y)=0 ; \quad R_{(n / 2)+1}(l / n, y)=\alpha \cdot y .
$$

The function $B_{0}(y)$ is a linear function of the coordinates $y$ according to the equation (10). The dependence of the $B_{0}(y)$ expressed in terms of stress, rather than through the angular movement.

It can be concluded from the relation (6), which in laminated beams, as in the continuous beams, tensions adjustment section is distributed linearly. Then the function $B_{0}(y)$ can be represented:

$$
B_{0}(y)=C \cdot y
$$

where $C$ - the coefficient of proportionality.

We determine its meaning, assuming that the bending moment along the length of the beam is constant: 


$$
\begin{aligned}
& 2 E \cdot h \cdot \sum_{i=1}^{n} \int_{0}^{H / 2} \frac{\partial R_{i}}{\partial x} \cdot y \cdot d y= \\
& =2 E \cdot h \cdot \int_{0}^{H / 2} n \cdot C \cdot y^{2} \cdot d y=\frac{h \cdot n \cdot H^{2}}{6} \cdot \sigma_{0},
\end{aligned}
$$

where $\sigma_{0}$ - the maximum value of the bending stress in the continuous bar in the same section as laminated beams, and the same value of the bending moment. Then:

$$
C=\frac{2 \sigma_{0}}{E \cdot H} \text {. }
$$

The shear stresses in the insulating layer will be the greatest in the corners of the steel sheets, [8]:

$$
\begin{aligned}
& \tau_{\max }^{\prime}=-\frac{G}{\delta} \cdot\left[R_{1}(0)-R_{n}(0)\right] ; \\
& \tau_{\max }^{\prime /}=-\frac{G}{\delta} \cdot\left[R_{1}(0)-R_{2}(0)\right] .
\end{aligned}
$$

If we assume that the relative displacement along the $x$ axis for the two points of outer surface of located at a distance $l$ from one another for a continuous beam at $y=H / 2$ is:

$$
\Delta R_{0}=\frac{\sigma_{0} \cdot l}{E},
$$

then laminated to the bar is the offset that can be presented subject to the availability of the axial displacement of the joints of two successive sheets in a layer (the first three summands), and the presence of the relative displacement of the edges of the sheet (last summand):

$$
\begin{aligned}
& \Delta R=2 R_{1}\left(0, \frac{H}{2}\right)-R_{2}\left(0, \frac{H}{2}\right)-R_{n}\left(0, \frac{H}{2}\right)+ \\
& +\sum_{i=1}^{n} \int_{0}^{l / n} \frac{\partial R_{i}\left(x, \frac{H}{2}\right)}{\partial x} .
\end{aligned}
$$

From equations (5), (6), (12) it follows that:

$$
\sum_{i=1}^{n} A_{i k}=0, \quad \sum_{i=1}^{n} B_{i k}=0 .
$$

Then on the grounds of (9) and (13):

$$
\sum_{i=1}^{n} \int_{0}^{l / n} \frac{\partial R_{i}\left(x, \frac{H}{2}\right)}{\partial x}=\int_{0}^{l / n} \frac{n \cdot \sigma_{0}}{E} \cdot d x=\frac{\sigma_{0} \cdot l}{E} .
$$

The ratio of $\Delta R / \Delta R_{0}$ characterizes increase flexural laminated steel bar compliance compared with a solid rod in the same cross-sectional. Consider the three schemes laying steel sheets lapped by $1 / 2,1 / 3$ and $1 / 4$ of the sheet (respectively, $n=2,3,4)$. Finally, we get:

$$
\begin{array}{r}
\text { 1) } n=2, \frac{\Delta R}{\Delta R_{0}}=1+\frac{4}{\lambda \cdot l}, \lambda=\sqrt{\frac{4 G}{E \cdot h \cdot \delta}}, \\
\left|\tau_{\max }^{\prime}\right|=\left|\tau_{\max }^{\prime \prime}\right|=\frac{2 G \cdot \sigma_{0}}{\lambda \cdot E \cdot \delta} ; \\
\text { 2) } n=3, \frac{\Delta R}{\Delta R_{0}}=1+\frac{3}{\lambda \cdot l}, \lambda=\sqrt{\frac{3 G}{E \cdot h \cdot \delta}}, \\
\text { 3) } n=4, \frac{\Delta R}{\Delta R_{\max }}=1+\frac{\tau_{\max }^{\prime \prime}}{\lambda}=\frac{3 G \cdot \sigma_{0}}{\lambda \cdot E \cdot \delta} ; \\
l \cdot\left(\lambda_{1}+2 \lambda_{2}\right)
\end{array}
$$




$$
\left\{\begin{array}{l}
R_{11}=-\frac{f \cdot p}{E \cdot h} \cdot x^{2}+C_{1} \cdot x+C_{2} ; \\
R_{14}=-\frac{f \cdot p}{E \cdot h} \cdot x^{2}+C_{1}^{\prime} \cdot x+C_{2}^{\prime} ; \\
R_{21}=\frac{f \cdot p}{E \cdot h} \cdot x^{2}+D_{1} \cdot x+D_{2} ; \\
R_{24}=\frac{f \cdot p}{E \cdot h} \cdot x^{2}+D_{1}^{\prime} \cdot x+D_{2}^{\prime} ; \\
R_{12}=A_{0}+B_{0} \cdot x+A_{1} \cdot \varepsilon^{\lambda x}+B_{1} \cdot \varepsilon^{-\lambda x} \\
R_{22}=A_{0}+B_{0} \cdot x-A_{1} \cdot \varepsilon^{\lambda x}-B_{1} \cdot \varepsilon^{-\lambda x} \\
R_{13}=A_{0}^{\prime}+B_{0}^{\prime} \cdot x+A_{1}^{\prime} \cdot \varepsilon^{\lambda x} \\
R_{23}=A_{0}^{\prime}+B_{0}^{\prime} \cdot x+A_{1}^{\prime} \cdot \varepsilon^{\lambda x}+B_{1}^{\prime} \cdot \varepsilon^{-\lambda x} \\
\lambda=\sqrt{\frac{4 G}{E \cdot h \cdot \delta}}
\end{array}\right.
$$

where $\varepsilon-$ the elastic deformation of the sample, which causes a voltage (it equals the ratio of change of the sample size after deformation to its initial size).

Most commonly the relationship between stress and deformation is linear (Hooke's law): $\sigma=\varepsilon \cdot E$.

The values of the coefficients of equations (19) can be obtained from the condition that the deformation is symmetrical, using the boundary conditions (20) and the conditions at the zones boundaries of sliding (21):

$$
\begin{gathered}
\left.\frac{\partial R_{23}}{\partial x}\right|_{x=0}=\left.\frac{\partial R_{21}}{\partial x}\right|_{x=0}=0 ; \\
\left.\frac{\partial R_{14}}{\partial x}\right|_{x=\frac{l}{2}}=\left.\frac{\partial R_{13}}{\partial x}\right|_{x=\frac{l}{2}}=0 ; \\
\left.R_{11}\right|_{s 1}=\left.R_{12}\right|_{s 2} ;\left.\quad R_{12}\right|_{s 2}=\left.R_{14}\right|_{s 2} ; \\
\left.R_{21}\right|_{s 1}=\left.R_{22}\right|_{s 1} ;\left.\quad R_{22}\right|_{s 2}=\left.R_{24}\right|_{s 2} \cdot \\
\left.\frac{\partial R_{11}}{\partial x}\right|_{s 1}=\left.\frac{\partial R_{12}}{\partial x}\right|_{s 1} ;\left.\frac{\partial R_{12}}{\partial x}\right|_{s 2}=\left.\frac{\partial R_{14}}{\partial x}\right|_{s 2} ; \\
\left.\frac{\partial R_{21}}{\partial x}\right|_{s 1}=\left.\frac{\partial R_{22}}{\partial x}\right|_{s 1} ;\left.\frac{\partial R_{22}}{\partial x}\right|_{s 2}=\left.\frac{\partial R_{24}}{\partial x}\right|_{s 2} ; \\
-\left.\frac{G}{\delta} \cdot\left(R_{11}-R_{21}\right)\right|_{s 1}=f \cdot p ; \\
-\left.\frac{G}{\delta} \cdot\left(R_{14}-R_{24}\right)\right|_{s 2}=f \cdot p .
\end{gathered}
$$

where $\alpha$ - angular deformation (rotation angle of a bar cross-sections at a height $y$ from the middle cross section), degrees:

$$
\begin{aligned}
& \alpha \cdot y=\frac{f \cdot p_{0}}{E \cdot h} \cdot\left(l+2 x_{1}\right) \cdot x_{1}- \\
& -\frac{f \cdot p_{0} \cdot \delta \cdot \lambda \cdot\left(\varepsilon^{\lambda x_{1}}-\varepsilon^{\lambda \cdot \frac{l}{2}} \cdot \varepsilon^{-\lambda x_{1}}\right)}{2 G \cdot\left(\varepsilon^{\lambda x_{1}}+\varepsilon^{\lambda \cdot \frac{l}{2}} \cdot \varepsilon^{-\lambda x_{1}}\right)} \cdot\left(\frac{l}{2}+2 x_{1}\right)+ \\
& +\frac{f \cdot p_{0} \cdot \delta}{G} \approx \frac{f \cdot p_{0}}{E \cdot h} \cdot\left(l+2 x_{1}\right) \cdot x_{1}+ \\
& +\frac{f \cdot p_{0} \cdot \delta}{G} \cdot\left(1+\frac{\lambda \cdot l}{4}+\lambda \cdot x_{1}\right) \\
& x_{2}=\frac{l}{2}-x_{1} .
\end{aligned}
$$

From the side of the equation (22), which is indicated by $(\approx)$, we will find:

$$
x_{1}=-\frac{\lambda \cdot l+4}{4 \lambda}+\sqrt{\frac{\lambda^{2} \cdot l^{2}-16}{16 \lambda^{2}}+\frac{2 G}{f \cdot p_{0} \cdot \delta \cdot \pi^{2}} \cdot \alpha \cdot y} .
$$

From here the maximum width the zone of sliding $\alpha$ is determined depending on the pressing pressure $p_{0}$ and the amount of the angular deformation bend of laminated element for a specific material, whose parameters are given values of $E, \mathrm{G}, f$ and dimensions of the steel sheets $(H, h, \delta)$ :

$$
\begin{aligned}
& b_{s}=x_{1} \cdot\left(\frac{H}{2}\right)=-\frac{\lambda \cdot l}{4 \lambda}+ \\
& +\frac{1}{\lambda} \cdot \sqrt{\frac{\lambda^{2} \cdot l^{2}-16}{16 \lambda^{2}}+\frac{G \cdot H}{f \cdot p_{0} \cdot \delta} \cdot \alpha} .
\end{aligned}
$$

Let us substitute into the equation (23) the value of $x_{1}=0$ to obtain the coordinates of the lower point of the zone of sliding $y_{0}$ (Fig. 2):

$$
y_{0}=\frac{f \cdot p_{0} \cdot \delta}{\alpha \cdot G} \cdot\left(1+\frac{\lambda \cdot l}{4}\right)
$$

If $y_{0} \geq h / 2$, that the sliding of sheet is virtually absent, and angular deformation $\alpha$ can be determined in this case as:

$$
\alpha=\alpha_{n}=\frac{2 f \cdot p_{0} \cdot \delta}{G \cdot H} \cdot\left(1+\frac{\lambda \cdot l}{4}\right),
$$

where $\alpha_{n}$ - angular deformation (rotation angle of the $n$-th plate in a section of the rod at the height $y$ from the middle cross section), degrees.

We introduce auxiliary coefficients:

$$
a=\frac{2 G \cdot H}{f \cdot p_{0} \cdot \delta \cdot \lambda^{2}}, \quad b=\frac{\lambda^{2} \cdot l^{2}}{16 \lambda^{2}} .
$$

The total bending moment $M(\alpha)$ for the two sections of adjacent sheets does not depend on the position of the point of consideration (does not depend on the coordinate $x$ ).

Moment depends only on the angular deformations $\alpha$ :

$$
\begin{aligned}
& M(\alpha)=2 h \cdot E \cdot \int_{0}^{H / 2}\left(\frac{\partial R_{1}}{\partial x}+\frac{\partial R_{2}}{\partial x}\right) \cdot y \cdot d y= \\
& =4 h \cdot E \cdot\left(\int_{0}^{y_{2}} B_{0}^{\prime} \cdot y \cdot d y+\int_{y_{0}}^{H / 2} B_{0} \cdot y \cdot d y\right)= \\
& =\frac{8 h \cdot E \cdot \lambda}{3(4+\lambda \cdot l)} \cdot \alpha \cdot y_{0}^{5}-f \cdot l \cdot\left(\frac{H^{2}}{4}-y_{0}^{2}\right)+ \\
& +\frac{16 f \cdot p_{0}}{15 \alpha^{2}}(3 a \cdot y-2 b) \cdot \sqrt{(b+a \cdot y)^{3}} \mid \begin{array}{l}
H / 2 \\
y_{0}
\end{array} .
\end{aligned}
$$

For continuous a bar of the same dimensions:

$$
M_{0}=\frac{E \cdot h \cdot H^{3}}{3 l} \cdot \alpha_{0} .
$$

If necessary to consider only the $\alpha$ and the angular deformation of the system there is no relative sliding, the bending moment in the angular area of deformations can be calculated:

$$
M(\alpha)=\frac{E \cdot h \cdot \lambda \cdot H^{3}}{3(4+\lambda \cdot l)} \cdot \alpha .
$$

The relative increase in the flexural suppleness laminated element can be calculated as the ratio of $M(\alpha)$ to 
$M_{0}$ (based on the presence of the insulation between the sheets and the possible the relative sliding of the sheets):

$$
\frac{\alpha}{\alpha_{0}}=\frac{E \cdot h \cdot H^{3} \cdot \alpha}{3 l \cdot M(\alpha)} \text {. }
$$

If in (30) substitute the value of $M(\alpha)$ from (29), the relative change in the flexural compliance laminated element can be represented by:

$$
\frac{\alpha}{\alpha_{0}}=1+\frac{4}{\lambda \cdot l} \text {. }
$$

Therefore, the relative change in the flexural compliance laminated element does not depend on $\alpha$.

\section{Conclusions:}

1. The results of the calculation of the flexural deformation of the laminated core of turbogenerator based on the compliance of the insulating cover sheet active steel are obtained. These calculations allow making a conclusion that the flexural rigidity of the core in the glued cores of turbogenerators decreases slightly, no more than $3 \%$. Therefore the modulus of elasticity of glued laminated of the core is reduced by no more than $3 \%$ compared to module of monolithic steel. In the not-bonded cores in which interconnection is provided only the pressing of sheets, stiffness decreases with increasing the flexural deformation.

2. The selection of the compaction pressure of the laminated core should be carried out based on the possible slip of sheets when vibration occurs, also taking into account knowledge of strength the adhesive layer and safe limits of relative sliding. Correctly selected compression effort reduces the relative sliding of the sheets in the package:

- the relative sliding of the laminated core sheets exists on the width not more than $2 \mathrm{~mm}\left(b_{S}<2 \mathrm{~mm}\right)$ in the joint area when pressure of pressing is $p_{0} \geq 1 \mathrm{MPa}$ and a amplitude of radial oscillations of vibration that do not exceed $A \leq 20 \mathrm{mkm}$. The effect of relative sliding sheet decreases a little the bending stiffness of the core in this case, not more than $1 \%$ :

- the relative sliding of the core sheets is practically absent in this range of vibrations and with compacting pressure $p_{0} \geq 1,5 \mathrm{MPa}$. Accordingly, weakening the pression in the core leads to an increase of vibration, to a reduce of the bending rigidity, to a reduce of the bending rigidity, to an increase of the relative sliding sheets (up to $10 \mathrm{~mm}$ ), to an abrasion of insulating coating of sheet active steel, to the connection of adjacent sheets, to increased losses in the steel, to heat until the «fire in the steel». Gluing laminated core sheets reduces the impact of this phenomenon.

3. Due to the presence the struts of radial ventilation the uneven pressure distribution arises after pressing, which can be neglected. This can be done, because this unevenness may be compensated in the glued packages with the help of binder materials, a shift steel sheets in packs without gluing only in a narrow region near the joints sheets sufficiently wide range of pressures. Ventilation spacers which establish beside the joints of sheets support the local increase of the pressure and decrease the slip zone. If the spacers are installed far away from the joints of sheets, they reduce the bending stiffness of the core and they extend the relative sliding zone.

4. Wear insulating layer between sheets of laminated core can be neglected, because:

- during the exploitation the pressure pressing cores decreases, which reduces the intensity of wear of the insulating layer;

- in areas where an insulating layer is erased, the products of wear are not removed but accumulated, and continue to perform the insulation function;

- during an operating process, the lacquer polymerization process that isolates, is continuing, which increases gluing of active steel sheets, increases the bending stiffness, reduces the vibration.

5. As a result it can be concluded that the period of exploitation of turbogenerators may be not less than 50 years, and not 20-25 years as previously thought, [4, 5]. This conclusion can be fundamental in determining possibility of extending the time of exploitation of the turbogenerators.

\section{REFERENCES}

1. Shevchenko V.V. Methods of quantitative assessment of technical condition electrical equipment to determine the need for its rehabilitation or replacement. Collected materials of the Int. sci.-pract. conf. "Modern trends in the MRO. Diagnostics of equipment of mining and metallurgical and energy complexes (PBMCs)», 10-12 September 2015, pp. 39-42. (Rus).

2. Vasilev V.S., Johansen V.I. Experience and projects construction of deep modernization of powerful turbogenerators stator in the conditions of power. Electrical Appliances 2003: Abstracts of the conference. St. Petersburg, Electrosila, 2003. pp. 9-12. (Rus).

3. Shevchenko V.V., Minko A.N. Modernization of constructions domestic turbogenerators taking into account the requirements to maintain their competitiveness. Bulletin of NTU «KhPI», 2014, no.38(1081), pp. 146-155. (Rus).

4. Warsawskiy G.Y. Influence segments and laminating the stator iron on the magnetic vibration of electrical machines AC. St. Petersburg: Collected Electrosila, 1989, no.37, pp. 42-45. (Rus).

5. Vaskovskyi Yu.N., Shumilov Yu.A., Shtogrin A.V. Analysis of vibration-exciting axial forces in a powerful turbo-generator stator core. Electrical engineering \& electromechanics, 2009, no.2, pp. 21-26. (Rus). doi: 10.20998/2074-272x.2009.2.04.

6. Geller P.L., Burak A.M., Sinayuk S.L. Indicators of active stator turbogenerator packages strength steel, which are glued. Dynamics and strength of powerful turbo- and hydrogenerators. - Collected USRIE, 1987, pp. 9-14. (Rus).

7. Johansen V.I. The effect on the rigidity of the stator core turbogenerator pasting active steel. Leningrad: Collection «Methods for calculating the turbo- and hydrogenerators», 1975, no.95, p. 103. (Rus).

8. Johansen V.I., Storozhev V.D. Calculation of the mechanical characteristics of the bonded electrical steel packages. Leningrad: Collection Electrosila, 1976, no.31, pp. 102-105. (Rus).

Received 25.04.2016

\section{V.V. Shevchenko, Candidate of Technical Science, Associate} Professor,

National Technical University «Kharkiv Polytechnic Institute», 21, Kyrpychova Str., Kharkiv, 61002, Ukraine.

phone +38050 407845, e-mail: zurbagan_@mail.ru

How to cite this article:

Shevchenko V.V. Influence of manufacturing quality of laminated core on a turbogenerator exploitation term. Electrical engineering \& electromechanics, 2016, no.4, pp. 28-33. doi: 10.20998/2074-272X.2016.4.04. 